\title{
Environmental Factors Affecting Situation Awareness in Unmanned Aerial Vehicles
}

\author{
Prasanna Velagapudi*, Sean Owens ${ }^{\dagger}$, Paul Scerri ${ }^{\ddagger}$ and Katia Sycara ${ }^{\S}$ \\ Carnegie Mellon University, Pittsburgh, PA, 15213, USA \\ Michael Lewis \\ University of Pittsburgh, Pittsburgh, PA, 15213, USA
}

\begin{abstract}
As mini-UAVs become more capable and reliable, it is important to start looking at the factors differentiating them from other classes of unmanned vehicles. One such factor is the physical proximity of operators to the vehicle during deployment. Operators of these UAVs are often within sight of their vehicle, and share many environmental cues such as visual landmarks. However, operating in the field also entails additional environmental stresses, such as less optimal use of computer equipment, variations in weather, and the physical demands of the terrain. In this paper, a pilot study is conducted to determine if any of these factors significantly impact situation awareness, by comparing operator performance in a visual identification task in a live field test with operators performing an identical task in a lab environment. Metric results suggest that performance is similar across the two conditions, but qualitative responses from participants suggest that the underlying strategies employed differ in the two conditions.
\end{abstract}

\section{Introduction}

$\mathrm{T}^{\mathrm{N}}$ recent years, there has been a surge in the application of unmanned aerial systems to a wide variety of problems. One particular class of vehicle that has been gaining favor in the community has been the fixed-wing mini-UAV. Small, low-cost, and portable, they are ideal for military and civilian applications ranging from short-range surveillance ${ }^{1}$ to environmental surveys ${ }^{2,3}$ to wilderness search-and-rescue. ${ }^{4}$

One interesting aspect of these vehicles is that their deployment model differs greatly from their larger, longer range counterparts. Specifically, many scenarios for mini-UAV deployment involve short-range missions, in which the UAV is deployed near (within a few miles of) the operator. In these cases terrain features, lighting, weather conditions, and even noises or sightings of the vehicle itself provide additional cues to human operators that may generally assist in interpreting data from the UAV. On the other hand, additional cognitive and physical costs are incurred by an operator making use of these cues, potentially increasing workload and decreasing performance. For instance, the operator may be required to physically turn to face the direction of the UAV, diverting attention away from a monitor showing a video feed, and requiring a subsequent mental context switch.

In order to interpret data from a UAV and make decisions about actions based on the data, the operator must maintain situation awareness. ${ }^{5}$ Put simply, if the operator's situation awareness is good, they can understand the context in which the UAV is operating and can interpret data coming from it more accurately and quickly. Like other forms of telepresence, UAV operators lack a number of potent cues, such as inertial feedback and peripheral vision, thus degrading their overall situation awareness. In an extreme example, a soldier using a mini-UAV in a hostile tactical environment might detect an opponent in its video stream but take minutes to work out the position of the opponent relative to themselves. Situation awareness has been well studied in the field of telerobotics, especially in applications pertaining to search and rescue ${ }^{6-8}$

*Ph.D. Candidate, Robotics Institute, pkv@cs.cmu.edu.

${ }^{\dagger}$ Research Programmer, Robotics Institute.

${ }^{\ddagger}$ Systems Scientist, Robotics Institute.

$\S$ Research Professor, Robotics Institute.

๑Professor, Department of Information Sciences \& Telecommunications. 
and the military. ${ }^{9,10}$ However, little work has looked at how the additional cues available to operators when co-located with a UAV in some environment impact situation awareness. Intuitively, being able to see and hear the UAV and its position relative to features in the environment should improve situation awareness because these extra cues are being provided in a very natural and familiar way. However, interpreting these cues and relating them back to a map entails additional cognitive load. It is not known whether the benefits of incorporating these cues outweigh the extra cognitive load when maintaining situation awareness.

There is a significant body of literature dealing with the effects of various user interfaces on situation awareness on UAV platforms. Drury et al. presents a decomposition of the factors that constitute humanUAV situation awareness, breaking the problem into those of maintaining spatial relationships, knowledge of vehicle intent and trust, and relevant environmental state. ${ }^{11}$ In the case of mini-UAVs, these issues are exacerbated by the very same characteristics that make them desirable. Low power and weight limits constrain sensing payloads to small sensors such as lightweight electro-optics, noisy GPS, and drifting inertial measurement. Operators are often limited to pose updates and streaming video of a small area of the ground. They must then use this information to identify targets, plan future trajectories and generally understand the situation on the ground. Furthermore, portability constraints typically limit the user interface to computing platforms that match the portability of the aircraft, such as laptops, PDAs, and embedded computing, limiting display and control hardware real-estate.

Much previous work has also focused on optimizing the designs of human-computer interfaces within these constraints to maximize operator situation awareness. Goodrich and colleagues have developed numerous such interfaces, from 3D projective overlays ${ }^{12,13}$ to PDA clients. ${ }^{14}$ Their work has focused mainly on presenting information from the UAV to the operator in a more natural way, rather than examining or exploiting the impact of the natural environment on task performance. In studies of UAV operator performance in the lab and in field trials for Wilderness Search and Rescue (WiSAR) tasks, Goodrich et al. acknowledge and account for the additional workload of field operators by having operators perform secondary tasks in lab trials. ${ }^{15}$ However, the potential beneficial impact of environmental cues and the actual difference in workload between these conditions is not directly studied.

The question addressed in this paper is whether the additional information available to an operator colocated with the UAV outweighs the cognitive and physical costs of using that information. By comparing the behavior and performance of UAV operators in the field against operators in lab environments lacking environmental cues, it is possible to analyze these effects on overall mission performance. Operators are given a visual search task in a wilderness environment, an activity which requires them to maintain 3D spatial relationships between the UAV and external targets. This spatial awareness is a core component of human-UAV situation awareness as described by Drury et al. ${ }^{11}$ We originally hypothesized that the additional cues available when the user co-located in the environment would aid situation awareness more than the additional cognitive and physiological load would degrade performance. We also anticipated that operators would be comfortable using environmental cues, hence the cognitive impact of using them would be relatively light. Instead, we found that performance did not vary significantly between these conditions, and that the field environment was found by participants to require slightly more effort to operate within.

\section{Method}

Experiment participants searched for colored targets using real-time video and telemetry data captured from a single mini-UAV flying a fixed search pattern over an outdoor test environment. They were tasked with marking these targets on a map of aerial overhead imagery. However, the environment in which the participants were situated was varied between a field condition and a lab condition. The interface hardware setup was replicated between both situations to ensure that user interactions were as similar as possible. In order to ensure that the two conditions were comparable despite the numerous variables affecting the live UAV flight patterns, data from each field test was logged and replayed in a corresponding lab trial.

\section{II.A. Platform}

The mini-UAV used in this study is the Procerus Unicorn platform, a 48" flying wing design with two fixed cameras, one looking forward and one looking to the right, both mounted at approximately a 30-degree downward angle. The cameras return an analog video signal with an effective resolution of approximately 648 x 486 pixels, as shown in Figure 2. In this experiment, only the forward-looking camera feed was captured. 


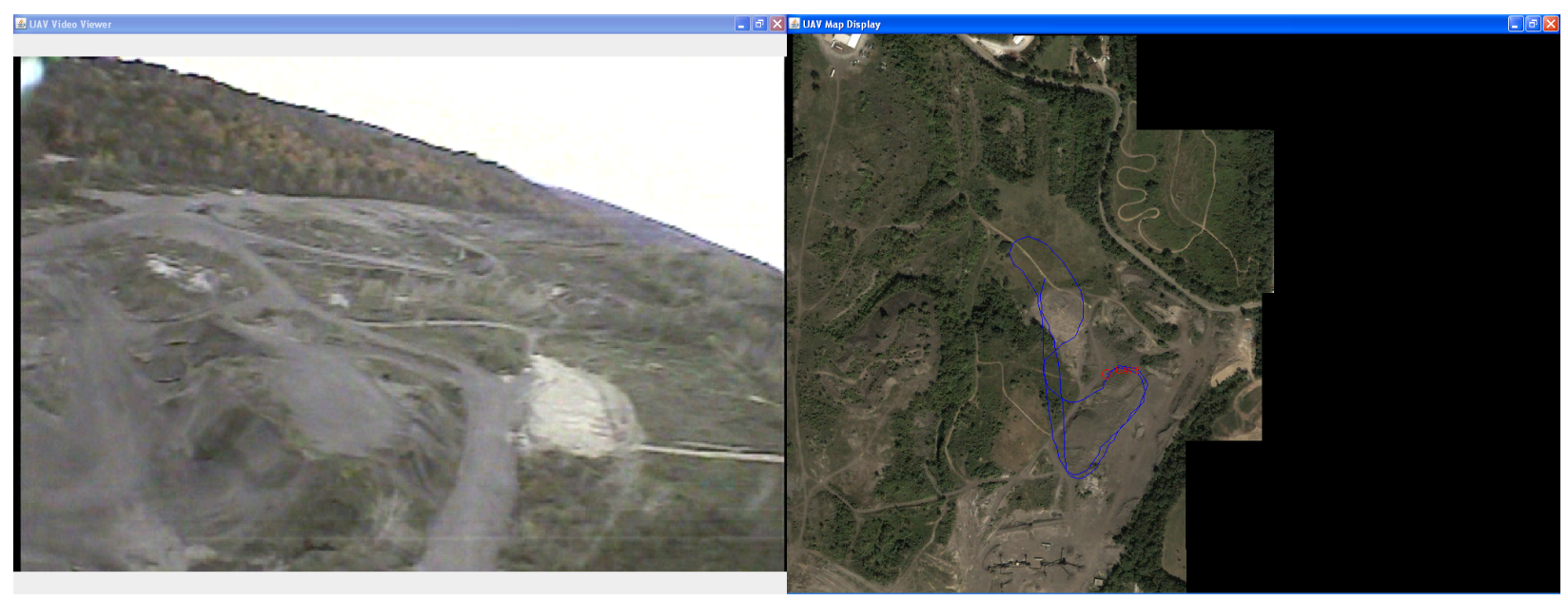

Figure 1. A dual display interface is used in which the left screen displays the video stream from the UAV while the right screen displays a map with aerial imagery of the test site overlaid with the GPS track of the UAV. Users see targets in the video stream on the left and mark their location on the map on the right.

The outdoor environment consisted of approximately two-hundred acres of partially wooded, uneven terrain. Targets were represented by orange fabric squares one meter on a side, and were not visible from the participants' location in the field condition. The UAV was flown in a waypoint loop at a constant speed of $14 \mathrm{~m} / \mathrm{s}$ at a fixed altitude of $60 \mathrm{~m}$ above ground. At this speed and altitude, targets in the camera feed were visible as small but distinctly colored objects that passed in and out of the field of view in several seconds. The GPS and video data was transmitted simultaneously to a ground control station on independent $900 \mathrm{Mhz}$ and $2.4 \mathrm{Ghz}$ channels, respectively. However, due to the low update rate of the GPS sensor and transmission delays, the two streams could be offset by up to a second.

\section{II.B. Interface}

The user interface, as seen in Figure 1, consisted of a single dual-display PC laptop connected via Ethernet to a UAV data server streaming either live or prerecorded data. On one screen, a video window displayed imagery from the forward camera of the UAV. On the other screen, a map window displayed an overhead aerial image of the test site. The current location of the UAV as measured by onboard GPS was plotted as a red circle on the map, and the history of previous locations was visible as a blue track. Within the map window, users could add, move, and remove targets using simple point-andclick mouse operations.

\section{II.C. Conditions}

Two conditions were tested, a field condition and a lab condition. In the field condition, participants completed the task using a live feed from a flying UAV at the test site. During this run, video and

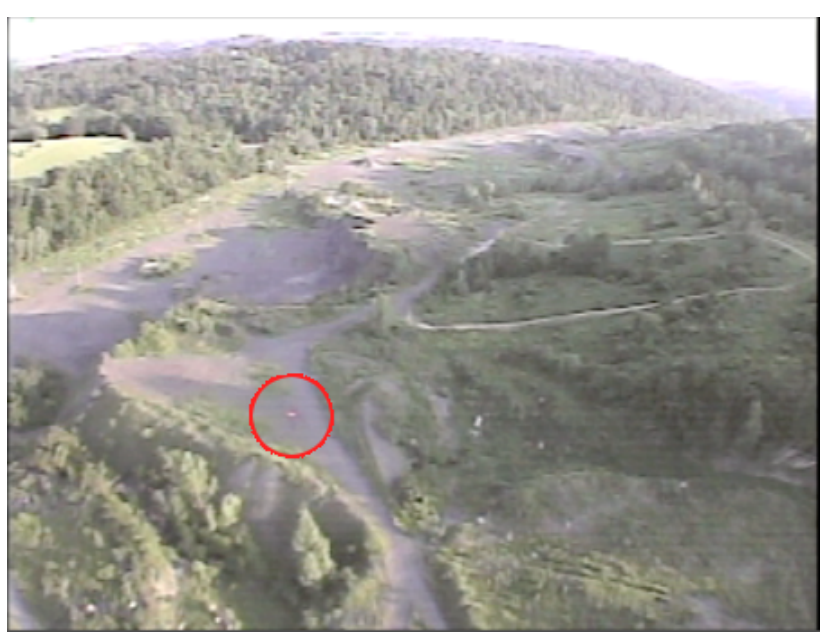

Figure 2. A UAV video sample containing the one meter square orange targets used in the experiment (circled in red). The target can be distinguished from the natural surroundings by its unique color.

GPS data from the UAV was logged to file. In the lab condition, participants completed the task in a controlled lab environment using data played back from from a corresponding field trial. The trials were organized in a randomized block design, in which trials are divided into blocks within which nuisance factors are held constant while a factor of interest is allowed to vary. This makes it possible to assess the effect of the factor of interest within blocks without having to worry about variations between blocks due to extraneous factors. In this case, the blocks were the individual UAV live flights. Since factors such as illumination 
and UAV trajectory could not be controlled precisely, each participant in the field condition was yoked to a participant in the lab condition. When the field trial was run, a complete data log was recorded for playback in the lab trial for the corresponding participant.

\section{II.D. Participants}

Ten robotics graduate students from within the department participated in this pilot study. Eight participants were male and two were female. Five participants were assigned to each condition. All of the subjects had prior experience with robots and were comfortable with the task.

\section{II.E. Procedure}

Participants first read written instructions on how to control the map interface. In both groups, participants were informed in the instructions that three targets were hidden in the environment. This was followed by a 5 minute training session in which prerecorded UAV GPS logs without video were replayed in the map interface and participants practiced adding, moving, and marking targets. Participants then performed a single 15 minute session in which they were presented with streaming video and GPS data and asked to locate the three targets. After the session, participants completed a NASA Task Load Index (TLX) ${ }^{16}$ and a brief questionnaire.

\section{Metrics}

Performance was assessed using several metrics of accuracy and speed. Markings were attributed to the nearest unique actual target. Marking error was calculated as the Euclidean distance in meters between the mark location and the corresponding target location. Since participants knew the correct number of targets, intermediate markers beyond the three closest were not included in the error calculation. In addition, participants often placed additional markers to indicate hypotheses of target locations, then used an elimination strategy in subsequent passes of the UAV, so these markings should not have been penalized. In every case, the final number of markers exactly equaled the number of targets.

The metric error of markers at the end of the run provided an initial measure of accuracy. This was obtained both as a cumulative total over all targets, and individually per target in 5 minute intervals throughout the run. This provided some measure of how quickly users were able to localize targets. However, while the yoking of participants would cancel the effects of variation in the UAV flight path, field of view, and target visibility, it did not compensate for the attention of participants. In situations where multiple targets were onscreen, it was possible that participants might focus on a single target and wait until the next UAV flyover to deal with other visible targets, propagating marker error further in time.

\section{Results and Discussion}

Taking advantage of the randomized block design, the aforementioned performance metrics were compared using two-way ANOVAs. Due to the small sample size, however, one-way ANOVAs were also performed over each condition group. There were no statistically significant differences under either measure for any of the metrics. However, there was an abnormally high variance for the final error of target 2 in the field condition, as seen in Figure 5, and slightly more pronounced reductions in error over the duration of the trials in the lab condition, as seen in Figure 4. It is unclear whether these effects were simply artifacts of high sample variance or subtle performance effects that were masked by the small sample size. Overall, the results of the field and lab performance metrics were statistically similar despite the differences in operator environment.

Measures of workload between the two groups were also statistically similar. A summary of the overall workload results is presented in Figure 3. Interestingly, participants in the field condition reported higher values on the TLX for effort than those in the lab condition. A two-way ANOVA yielded $p=0.093$, and a one-way ANOVA yielded $p=0.069$. This might suggest that while performance was not impaired, certain environmental factors were increasing operator workload.

In the post-experiment questionnaire, several interesting trends provided some insight into potential effects. Some characteristic excerpts are presented in Table 1. While the metric error was similar between the two conditions, the majority of participants in the field conditions believed that participants would 


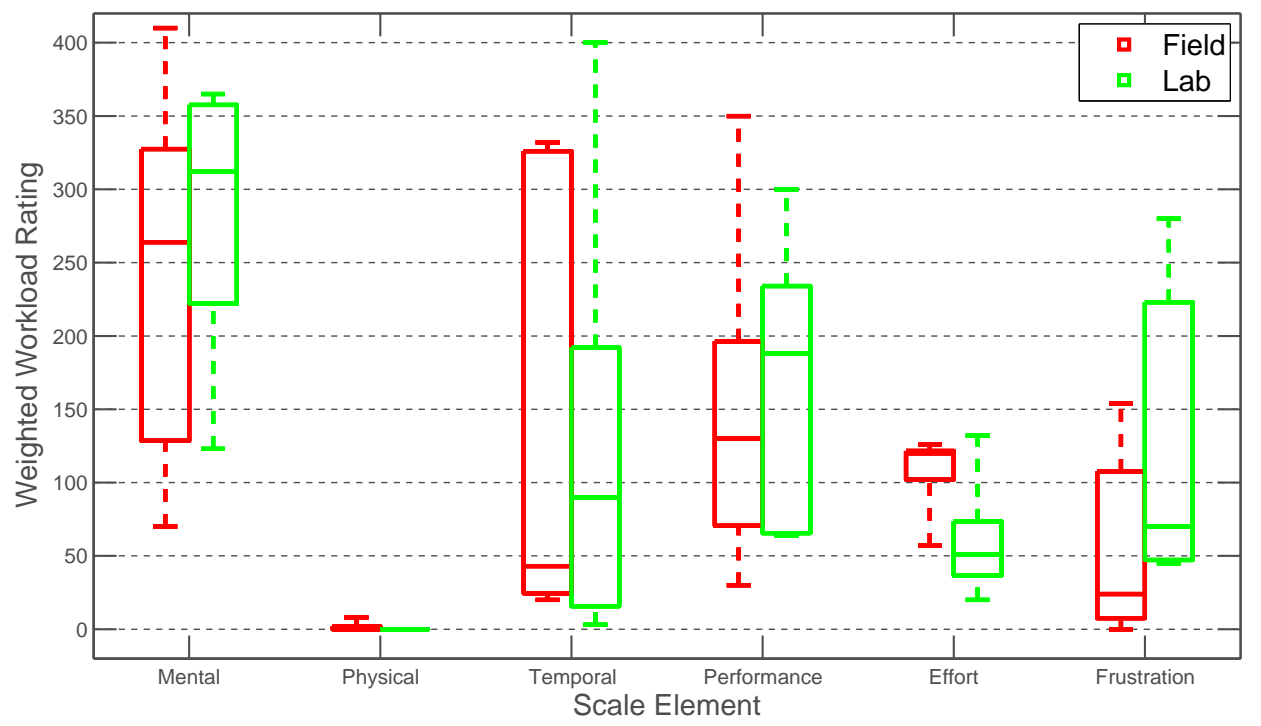

Figure 3. TLX workload ratings for the task were similar across both conditions, with the exception of the effort element. TLX ratings for effort were slightly higher in the field condition than in the lab condition (1-way ANOVA, $p=0.069$, 2-way ANOVA, $p=0.093)$.

perform better in a field condition than in a lab condition (4 out of 5), while only two participants in the lab group thought this would be the case. Participants in the lab condition reported a reliance on visual landmarks in the video, with 4 out of 5 specifically mentioning using road and trail intersections to help place markers. In the field condition, none of the participants mentioned the road network, but several mention using environmental cues to place markers. Two participants mentioned using terrain knowledge from being in the field to improve their estimate, one mentioned using their view of the terrain and the UAV to help solve for the camera bearing, and one participant reported using their own position in the UAV's video stream to help register objects in the UAV's field of view. In addition, one participant believed that glare on the two user displays had adversely impacted their performance, indicating that certain environmental factors might also be hindering performance. Interestingly, although the responses suggested that participants were using different strategies, none of these comments appeared to correspond to significant differences in performance.

Table 1. Excerpts from the questionnaire suggest that participants in the field condition were using environmental cues to perform the task, and that participants in the lab condition focused greatly on road networks.

\begin{tabular}{|l|l|}
\hline \multicolumn{1}{|c|}{ Field Condition } & \multicolumn{1}{c|}{ Lab Condition } \\
\hline $\begin{array}{l}\text { "Seeing [the ground station] when the UAV was flying } \\
\text { over helped me to estimate where with respect to the }\end{array}$ & "The roads were the most useful feature." \\
UAV is an object." & $\begin{array}{l}\text { "I primarily matched roads and paths, especially their } \\
\text { junctions to locate the scene on the map." }\end{array}$ \\
$\begin{array}{l}\text { "Just knowing the landscape beforehand helps } \\
\text { definitely." }\end{array}$ & \\
\hline
\end{tabular}

\section{Conclusion}

Contrary to the original hypotheses, there were few differences in performance or workload between the two conditions. While the sample sizes used in this pilot study were small, the similarities between the two conditions, coupled with the feedback from the participants, suggest that external cues and distractions neither strongly benefit nor impede operators in this visual search task. However, there are notable differences in the reported strategies of participants in the two groups, suggesting that while performance was similar in this case, there may be differences in underlying strategies. Since both advantageous and detrimental effects were reported, future experiments may need to isolate potentially confounding environmental factors. 


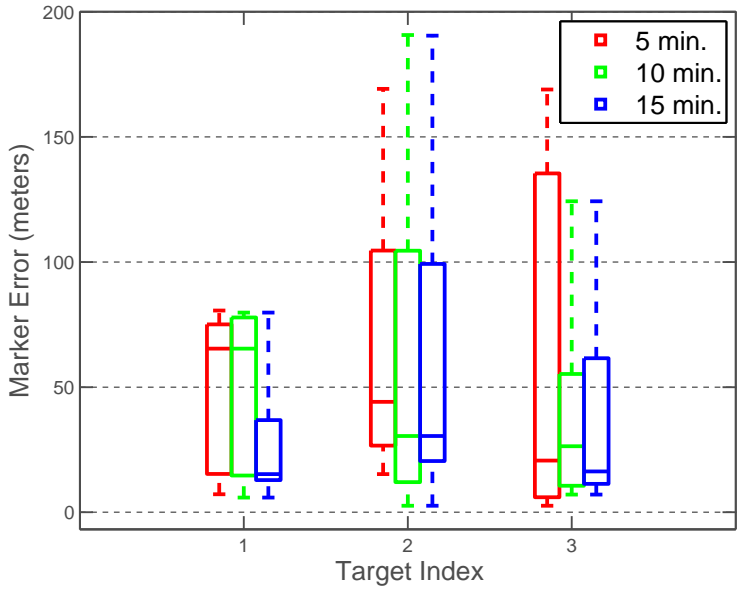

(a) Field Condition

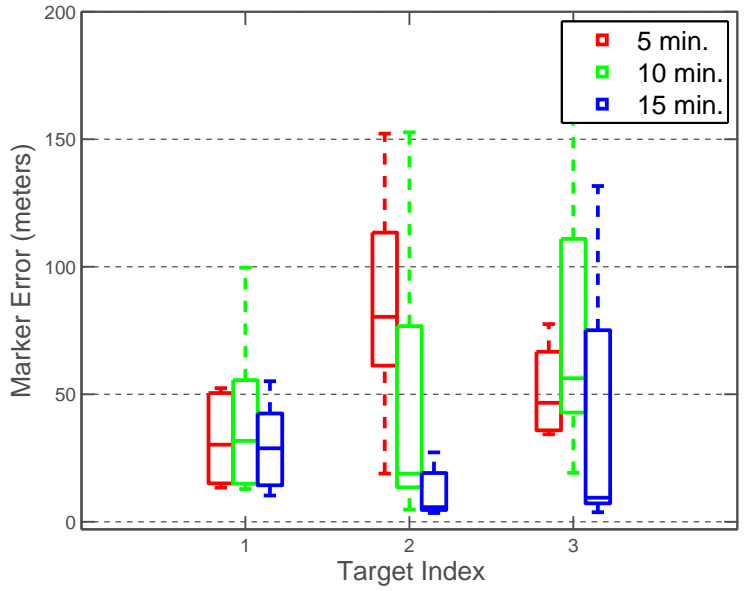

(b) Lab Condition

Figure 4. The error of the target markers decreased over time in both conditions, but the effect was most pronounced in targets 2 and 3 in the lab condition.

In addition, certain strategies may have been hindered by the experimental setup used in this experiment. The fast pace and timing of the task may have discouraged participants from the context switching necessary to move from the map interface to their local environmental view. In addition, several optical effects would discourage participants from focusing visual attention on environmental cues. In switching between the backlit display monitors and the sunlit outdoor environment, participants needed to shift their depth-of-focus and adjust to changes in ambient brightness. The physiological strain of this switch may have played a role in reducing the significance of environmental effects.

A future study of this task will address these specific factors independently, to help determine if any more subtle or complex effects exist that may influence performance or workload metrics. If these factors are found not to significantly affect operator execution of the task, it will help transfer the results of controlled lab experiments examining other effects on operators, such as the effects of more complex interface designs, ${ }^{4}$ to field systems. Given the relatively high cost of equipment and personnel to run mini-UAV field experiments, such a mapping is highly desirable for its potential to lower the barrier of entry to mini-UAV research.

\section{Acknowledgments}

This research has been funded in part by the AFOSR grant FA9550-07-1-0039 and the AFOSR MURI grant FA9550-08-1-0356. This material is based upon work supported under a National Science Foundation Graduate Research Fellowship.

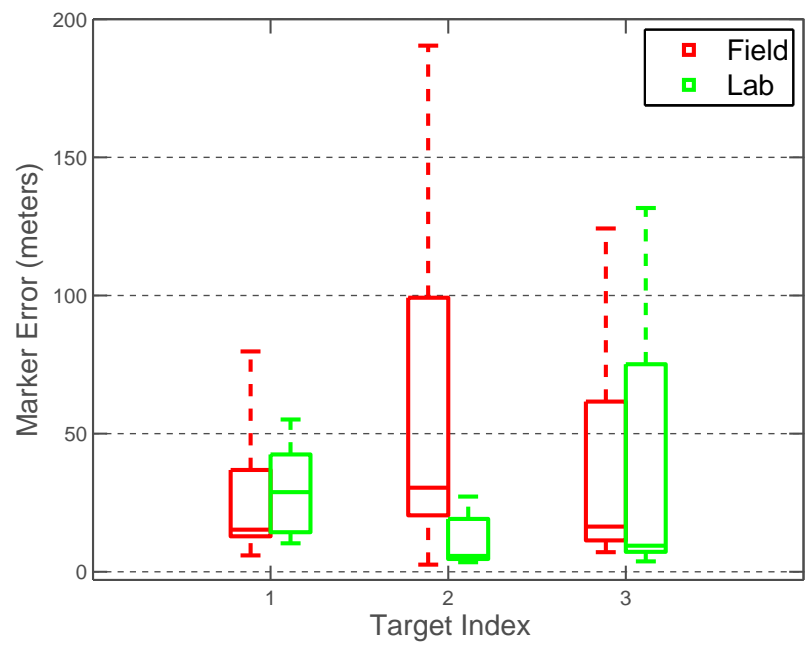

Figure 5. Marker error in meters for the three targets over the two conditions. While marker 2 had an unusally high variance, there was no strong statistically significant difference between the conditions (1-way ANOVA, $p=0.160$, ference between the cond
2-way ANOVA, $p=0.205$ ). 


\section{References}

${ }^{1}$ Coffey, T. and Montgomery, J., "The emergence of mini UAVs for military applications, ser. Defense Horizons," National Defense University: Center for Technology and National Security Policy, Vol. 22, 2002.

${ }^{2}$ Lee, K., Development of Unmanned Aerial Vehicle (UAV) for wildlife surveillance, Ph.D. thesis, University of Florida, 2004.

${ }^{3}$ Jones, B., An Evaluation of a Low-Cost UAV Approach to Noxious Weed Mapping, Ph.D. thesis, Brigham Young University, 2007.

${ }^{4}$ Goodrich, M., Cooper, L., Adams, J., Humphrey, C., Zeeman, R., and Buss, B., "Using a mini-uav to support wilderness search and rescue: Practices for human-robot teaming," Proceedings of the IEEE International Workshop on Safety, Security, and Rescue Robotics, 2007.

${ }^{5}$ Endsley, M., "Design and evaluation for situation awareness enhancement," Human Factors and Ergonomics Society Annual Meeting Proceedings, Vol. 32, Human Factors and Ergonomics Society, 1988, pp. 97-101.

${ }^{6}$ Riley, J. and Endsley, M., "Situation Awareness in HRI with Collaborating Remotely Piloted Vehicles," Human Factors and Ergonomics Society Annual Meeting Proceedings, Vol. 49, Human Factors and Ergonomics Society, 2005, pp. 407-411.

${ }^{7}$ Scholtz, J., Young, J., Drury, J., Yanco, H., and NIST, G., "Evaluation of human-robot interaction awareness in search and rescue," Proceedings of IEEE International Conference on Robotics and Automation, 2004.

${ }^{8}$ Murphy, R., "Human-robot interaction in rescue robotics," IEEE Transactions on Systems, Man, and Cybernetics, Part C: Applications and Reviews, Vol. 34, No. 2, 2004, pp. 138-153.

${ }^{9}$ Ross, B., Bares, J., Stager, D., Jackel, L., and Perschbacher, M., "An Advanced Teleoperation Testbed," Field and Service Robotics: Results of the 6th International Conference (STAR: Springer Tracts in Advanced Robotics Series Volume 42), 2008.

${ }^{10}$ Cummings, M., Nehme, C., Crandall, J., and Mitchell, P., "Predicting Operator Capacity for Supervisory Control of Multiple UAVs," Innovations in Intelligent Machines, 2007.

${ }^{11}$ Drury, J., Riek, L., and Rackliffe, N., "A decomposition of UAV-related situation awareness," Proceedings of the 1st ACM SIGCHI/SIGART conference on Human-robot interaction, 2006.

${ }^{12}$ Cooper, J. and Goodrich, M., "Towards combining UAV and sensor operator roles in UAV-enabled visual search," Proceedings of the 3rd ACM/IEEE international conference on Human-robot interaction, 2008.

${ }^{13}$ Drury, J. L., Richer, J., and Rackliffe, N., "Comparing situation awareness for two unmanned aerial vehicle human interface approaches," In Proceedings of the IEEE International Workshop on Safety, Security and Rescue Robotics, 2006.

${ }^{14}$ Quigley, M., Goodrich, M., and Beard, R., "Semi-autonomous human-UAV interfaces for fixed-wing mini-UAVs," Proceedings of the 2004 IEEE/RSJ International Conference on Intelligent Robots and Systems, 2004.

${ }^{15}$ Goodrich, M., Morse, B., Gerhardt, D., Cooper, J., Quigley, M., Adams, J., and Humphrey, C., "Supporting wilderness search and rescue using a camera-equipped mini UAV," Journal of Field Robotics, 2008.

${ }^{16}$ Hart, S. and Staveland, L., "Development of NASA-TLX (Task Load Index): Results of empirical and theoretical research," Human mental workload, Vol. 1, 1988, pp. 139-183. 